\title{
Brain donation in psychiatry: results of a Dutch prospective donor program among psychiatric cohort participants
}

\author{
Geertje M. de Lange ${ }^{1,2^{*}}$, Marleen Rademaker ${ }^{1}$, Marco P. Boks ${ }^{2}$ and Saskia J. M. C. Palmen ${ }^{2}$
}

\begin{abstract}
Background: Human brain tissue is crucial to study the molecular and cellular basis of psychiatric disorders. However, the current availability of human brain tissue is inadequate. Therefore, the Netherlands Brain Bank initiated a program in which almost 4.000 participants of 15 large Dutch psychiatric research cohorts were asked to register as prospective brain donors.

Methods: We approached patients with schizophrenia, bipolar disorder, major depressive disorder, obsessive-compulsive disorder, post-traumatic stress disorder, families with a child with autism or Attention Deficit Hyperactivity Disorder, healthy relatives and healthy unrelated controls, either face-to-face or by post. We investigated whether diagnosis, method of approach, age, and gender were related to the likelihood of brain-donor registration.

Results: We found a striking difference in registration efficiency between the diagnosis groups. Patients with bipolar disorder and healthy relatives registered most often (25\% respectively $17 \%)$, followed by unrelated controls (8\%) and patients with major depressive disorder, post-traumatic stress disorder, and obsessive-compulsive disorder (9\%,6\% resp. 5\%). A face-to-face approach was 1.3 times more effective than a postal approach and the likelihood of registering as brain donor significantly increased with age. Gender did not make a difference.

Conclusions: Between 2013 and 2016, our prospective brain-donor program for psychiatry resulted in an almost eightfold increase (from 149 to 1149) in the number of registered psychiatric patients at the Netherlands Brain Bank. Based on our results we recommend, when starting a prospective brain donor program in psychiatric patients, to focus on face to face recruitment of people in their sixties or older.
\end{abstract}

Keywords: Brain bank, Psychiatry, Post-mortem, Registration rates, Recruitment, Prospective brain-donor program

\section{Background}

The use of human brain tissue is the main strategy to study the molecular and cellular basis of psychiatric disorders. However, the current availability is far from sufficient: $69 \%$ of the post-mortem studies on bipolar disorder in the last 30 years used material from a single source consisting of merely 50 cases (Stanley Foundation Collection) and for the second most widely used collection (Harvard), $80 \%$ of studies were conducted with the same 72 cases [1]. Increasing the number of available post-mortem brains of extensively phenotyped patients

* Correspondence: G.M.deLange@umcutrecht.nl

${ }^{1}$ Netherlands Brain Bank, Netherlands Institute for Neuroscience, Meibergdreef 47, 1105, BA, Amsterdam, the Netherlands

${ }^{2}$ Brain Center Rudolf Magnus, University Medical Center Utrecht, Utrecht University, PO Box 85500, 3508, GA, Utrecht, the Netherlands will facilitate post-mortem research, and is key in improving understanding of the pathophysiology of psychiatric disorders. Ultimately, this should lead to improved treatment strategies, alleviating the personal, social, and economic burden of psychiatric disorders [2, 3]. Therefore, the Netherlands Brain Bank (NBB) initiated the first worldwide prospective donor program for seven psychiatric disorders (NBB-Psy). Its aim is to establish a resource of well documented and high-quality brain tissue of patients diagnosed with schizophrenia (SCZ), bipolar disorder (BD), major depressive disorder (MDD), obsessivecompulsive disorder (OCD), post-traumatic stress disorder (PTSD), autism spectrum disorder (ASD) and Attention Deficit Hyperactivity Disorder (ADHD), non-psychiatric relatives and unrelated control subjects. To achieve this, 
NBB-Psy, in close collaboration with five Dutch university medical centers, actively approached extensively phenotyped participants of fifteen large psychiatric research cohorts to register as prospective brain donor at the NBB. We here present an interim report of the registration percentages of patients, relatives and unrelated control subjects, taking into account the effects of diagnosis, method of approach, age, and gender.

We hypothesized registration rates of $15-20 \%$, which was based on our experience with approaching cohort patients with neurological disorders where the registration rates were 20 to $25 \%$. We expected the registration rates in psychiatry to be somewhat lower than in neurology. In neurological disorders confirming the diagnosis post-mortem is an additional motivational factor [4]. Moreover, patients are on average older, and their disease has a clear neurodegenerative nature, which confronts patients with increasing constraints on their daily function and may thus motivate to contribute to scientific studies to eradicate the disorder they suffer from. We hypothesized that older people would be more willing to register [5], and that a face-to-face approach would be more effective than a postal approach. Finally, we hypothesized that gender [5] and diagnosis would not affect registration rate.

\section{Methods}

\section{Study design and participants}

Our study was a quantitative descriptive study of participants of fifteen psychiatric research cohorts in the Netherlands. We requested 3751 cohort participants to consider registering for brain donation with NBB-Psy. This concerned patients with at least one of the seven following DSM-IV diagnoses: SCZ, BD, MDD, OCD, PTSD, ASD and ADHD, first-degree relatives, and healthy control subjects. Table 1 shows the number of approached participants per diagnosis group. As the OCD and PTSD cohort studies did not include any relatives, the number of relatives of OCD and PTSD patients approached is zero.

Participants of the ASD and ADHD cohorts were families of which the patients were minors. Within these cohorts we only approached the parents, since minors are not eligible for registration. Therefore, we cannot present registration rates of patients with ASD and ADHD.

Table 1 The number of approached patients, relatives and controls

\begin{tabular}{lllllll}
\hline & SCZ & BD & MDD & OCD & PTSD & Controls \\
\hline Patients & 39 & 947 & 1139 & 493 & 265 & \\
Relatives & 105 & 262 & 64 & 0 & 0 & \\
Controls & & & & & & 465 \\
\hline
\end{tabular}

\section{Procedures}

With the principal investigator (PI) of each cohort study, we agreed to inform all adult cohort participants individually about NBB-Psy by means of a letter from the PI explaining the collaboration with NBB-Psy. We attached the NBB-Psy brochure, which describes why brain research of psychiatric disorders is crucial, and what to expect at the time of death. We approached as many people as possible face-to-face, immediately following their visit for the cohort study. If face-to-face contact was not possible, we approached them by post. After the first approach, we contacted participants within two weeks by telephone to offer further information unless participants objected. If the participant was interested in registering as brain donor, we sent extended information and registration forms. These forms are to be signed not only by the prospective brain donor, but also by a nextof-kin. Although Dutch law does not require permission of a next-of-kin, the NBB requires it for two reasons. First, in order to prevent someone who is (temporarily) incompetent to make the decision of becoming brain donor completely by himself. Second, co-signature ensures that people close to the prospective brain donor are informed about the registration, which increases the chance that they will contact the NBB when the brain donor dies. After three months, if the participant had not registered yet, we made a reminder phone call.

For this study, we analyzed data regarding the cohort participants who were approached between August 1, 2013 and July 1, 2015. As there was a delay of, on average, 105 days between first approach and date of actual registration, we assessed the number of registrations of these participants until October 14, 2015. All NBB-Psy procedures were approved by the medical ethics committee.

\section{Diagnosis}

In order to analyze the relation of diagnosis with the registration rate, every cohort participant was categorized as either one of the seven NBB-Psy disorders, a relative or a control subject. In all cohort studies, the diagnoses of participants were confirmed by trained research assistants using one or multiple of the following instruments: Structured Clinical Interview for DSM-IV (SCID-I), Mini International Neuropsychiatric Interview (M.I.N.I.) plus, Comprehensive Assessment of Symptoms and History (CASH), or Composite International Diagnostic Interview (CIDI).

\section{Statistical analysis}

Statistical analyses were carried out using SPSS 22.0. We assessed the registration percentages per group (SCZ, $\mathrm{BD}, \mathrm{MDD}$, OCD, PTSD, relatives, controls). We investigated whether age, gender, method of approach, and 
diagnosis affected brain-donor registration percentages. One single logistic model was used with dummy coding for the diagnostic groups relative to the controls with 'registered as brain donor' (yes or no) as outcome in order to compare: 1 . BD versus controls, 2 . MDD versus controls, 3. OCD versus controls, 4. PTSD versus controls, and 5. healthy relatives versus controls. The group SCZ was too small $(n=39$ approached and $n=1$ registered) to be included in the analyses. Approach method (face-to-face versus post) was a determinant and age and gender covariates. Assumptions of homogeneity of variance were checked.

We used the forward stepwise conditional method as implemented in SPSS to find the best predictive model for brain-donor registration.

\section{Results}

\section{Demographic measures}

We approached 3751 cohort participants. Ninety-five percent $(n=3567)$ accepted the NBB-Psy brochure (info1). Thirty-nine percent $(n=1446)$ of all the approached participants expressed a positive attitude towards brain donation and were willing to receive detailed information and registration forms (info2). Thirteen percent $(n=485)$ of the approached cohort participants registered as brain donor. During the period under study (August 2013 - October 2015), three participants withdrew their consent and five passed away and underwent autopsy. Table 2 shows the numbers for each psychiatric disorder.

Two hundred and four participants were excluded from the analyses: SCZ patients were excluded as a group because the number of SCZ participants approached $(n=39)$ and registered $(n=1)$ was too low; of $4 \%$ of the participants $(n=141)$ age was unknown and $0.6 \%(n=24)$ of the participants actively approached us (therefore, method of approach could not be analyzed). Exclusion of these subjects resulted in a sample of 3547 participants included in the analysis.

\section{Group and likelihood of registration}

The likelihood of a patient with BD to register as a brain donor was 3.70 times higher than that of a control subject $(\beta=1.309, \mathrm{~W}=42.81, p<0.001)$. For a healthy relative the likelihood was 2.04 times higher $(\beta=0.710$, $\mathrm{W}=9.62, p=0.002)$. The likelihood of brain-donor registration for $\mathrm{MDD}(\beta=-0.067, \mathrm{SE}=0.20$, $\mathrm{W}=0.11, p=0.74), \mathrm{OCD}(\beta=-0.284, \mathrm{SE}=0.27$, $\mathrm{W}=1.08, p=0.30)$ and $\operatorname{PTSD}(\beta=-0.397, \mathrm{SE}=0.42$, $\mathrm{W}=0.92, p=0.34)$ patients was similar to control subjects. See Fig. 1.

\section{Age and likelihood of registration}

The likelihood of someone registering as a brain donor increased with age at least until the age of $70(\beta=0.024$, $\mathrm{W}=40.00, p<0.001)$. Figure 2 shows the registration percentages by age group.

\section{Gender and likelihood of registration}

There was no effect of gender on the likelihood of registering as brain donor: $(\beta=-0.007, \mathrm{~W}=0.004, p=0.95)$.

\section{Approach method and likelihood of registration}

Participants who were approached face to face registered 1.30 times more often compared to participants

Table 2 the approach of cohort participants, by disorder

\begin{tabular}{|c|c|c|c|c|c|c|c|c|}
\hline Disorder & Cohort & Approached (n) & Average age (y) & Male & Accepted info1 & Permission to phone & Info2 & Registered \\
\hline SCZ & GROUP & 39 & 37 & $82 \%$ & $n / a^{b}$ & $100 \%$ & $23 \%$ & $3 \%$ \\
\hline $\mathrm{BD}$ & $\mathrm{BiG}, \mathrm{DIADE}$ & 947 & 53 & $46 \%$ & $99 \%$ & $95 \%$ & $59 \%$ & $25 \%$ \\
\hline MDD & NESDA, NESDO, ECT & 1139 & 54 & $31 \%$ & $90 \%$ & $84 \%$ & $37 \%$ & $9 \%$ \\
\hline OCD & AMC regular \& DBS, NOCDA & 493 & 48 & $46 \%$ & $95 \%$ & $82 \%$ & $21 \%$ & $5 \%^{c}$ \\
\hline PTSD & BEPP/EMDR, BioMap, Booster, Paroxetine/CGT, PO & $265^{a}$ & 48 & $52 \%$ & $\mathrm{n} / \mathrm{a}$ & $57 \%$ & $16 \%$ & $6 \%$ \\
\hline Family & GROUP, BiG, NESDA & 403 & 61 & $45 \%$ & $100 \%$ & $94 \%$ & $39 \%$ & $17 \%$ \\
\hline Control & GROUP, BiG, NESDA, NESDO, Booster, DIADE & 465 & 53 & $39 \%$ & $92 \%$ & $88 \%$ & $32 \%$ & $8 \%$ \\
\hline Total & & 3751 & 53 & $41 \%$ & $95 \%$ & $86 \%$ & $39 \%$ & $13 \%$ \\
\hline
\end{tabular}

NESDA = The Netherlands Study of Depression and Anxiety. NESDO = The Netherlands study of Depression in the Elderly. ECT = Depressed patients treated with electroconvulsive therapy. BiG = Bipolar Genetics. DIADE = Diagnostic Imaging of Affective Disorders using Emotion Processing. GROUP $=$ Genetic Risk and Outcome in Psychosis. AMC regular = Patients who had a diagnostic intake or were treated for OCD at the Academic Medical Center in Amsterdam. AMC DBS = Patients with OCD who underwent deep brain stimulation at the AMC. NOCDA = The Netherlands OCD Association Study: identifying risk factors for chronicity in the course of obsessive-compulsive disorder. Booster = The effect of oxytocin on brain activity in police officers. Paroxetine/CGT = Randomized controlled trial 'the effectiveness of paroxetine versus trauma-focused cognitive behavioral therapy in the treatment of PTSD'. BioMap = The 'biological markers for PTSD' study. BEP/EMDR = 'Effectiveness and efficiency of Eye Movement Desensitization and Reprocessing therapy (EMDR) versus Brief Eclectic Psychotherapy (BEP) in the treatment of PTSD'. PO = Police officers who have been treated for PTSD at the Academic Medical Center

${ }^{\mathrm{a}} 30 \%(n=40)$ of BEPP/EMDR participants could not be reached by phone, possibly due to outdated contact information

${ }^{b} \mathrm{n} / \mathrm{a}=$ non applicable, because info1 was sent by post

'Those treated with Deep Brain Stimulation $(n=10)$ registered significantly more often (30\%) 


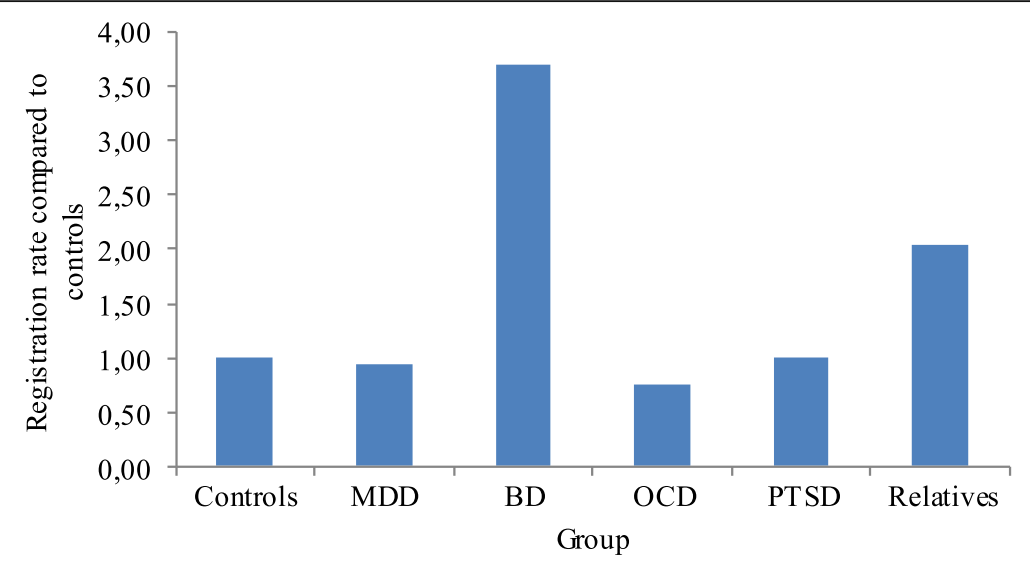

Fig. 1 Likelihood of registration per diagnosis, with healthy control subjects as comparison

approached by post, which was a significant difference $(\beta=0.25, \mathrm{~W}=3.90, p=0.048)$.

\section{Best predictive model for brain-donor registration}

The forward conditional method of logistic regression shows that the model, which best predicts the likelihood of a cohort participant registering as a brain donor includes the variables: age, method of first approach, and diagnostic group and explained around 10\% of the variation $\left(R^{2}\right.$ (Nagelkerke). $\left.=0.102\right)$.

\section{Discussion}

In the present study, we asked psychiatric cohort participants to consider brain donation and we investigated the influence of gender, age, diagnosis, and method of approach on the willingness to sign up as brain donor. The overall registration rate was $13 \%$, which is somewhat lower than we hypothesized (15-20\%), and indeed lower than those reported for neurological cohorts (31\% to $85 \%$ [6-9]). BD patients and relatives, however, did register at the anticipated rate (resp. $25 \%$ and $17 \%$ ). Surprisingly, they registered more often compared to all other groups, which may be due to the fact that BD patients often articulate that their illness causes high burden, and, combined with significant genetic load of the disorder, BD patients are eager to participate in (postmortem) research. Although our sample was small, within the OCD group, patients treated with DBS registered significantly more often than other patients with OCD. This may also be due to the high burden of this DBS group, who has the most severe and therapy-resistant form of OCD. In addition, DBS patients have electrodes in their brains, which may make the link to (postmortem) brain research more logical. We expect a similar mechanism in the ECT-depression cohort, which we recently started to recruit.

In line with our hypothesis, participants, initially approached face-to-face, registered significantly more often compared to those initially approached by post. This finding also fits well with previous studies [6-8].

The likelihood of someone registering as brain donor increased with age, which is in accordance with the results of Kaye and colleagues, who found that age was positively correlated to the rate of brain-donation consent among healthy elderly participants of a

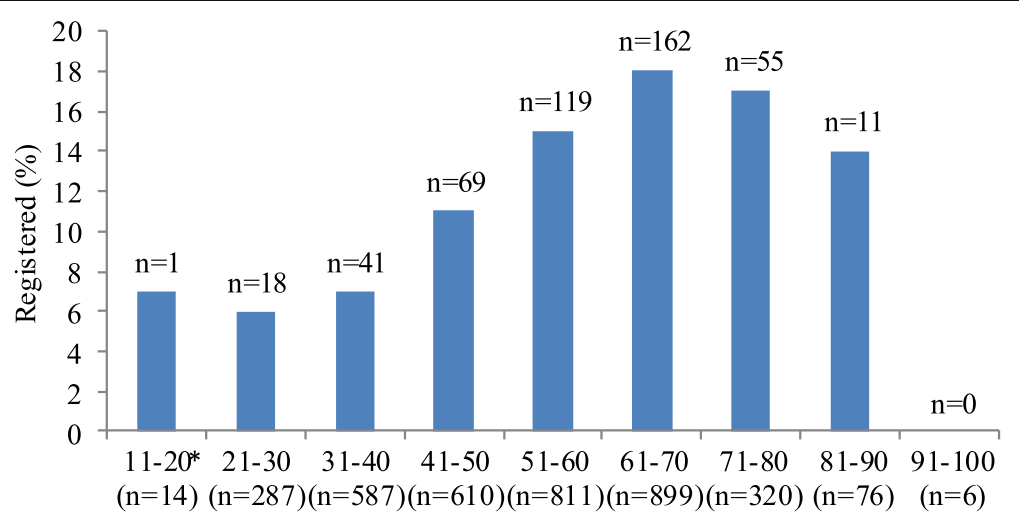

Age and number of approached cohort participants

Fig. 2 Percentage of cohort participants registered as brain donor, by age group. * Age 18-20 
longitudinal study on successful aging [5]. In absolute numbers, more females than males registered as brain donor, similar to an Australian brain donation program $[10,11]$. However, we approached more women than men, so the percentage of women and men who registered was not significantly different, which is in accordance with previous studies [5, 8]. A limitation of the present study is that we were not able to perform analyses on the SCZ group due to the small sample size, nor on relatives of OCD or PTSD patients, as they were not included in the cohort studies. In addition, we could not include ASD and ADHD in our analyses because we did not have data on adult cohort participants with these diagnoses. Finally, we note that the numbers presented here concern prospective brain donors. In the period under study, three registered cohort participants withdrew their consent on second notice. The experience of the NBB is that only very few people withdraw their consent. In addition, it hardly ever occurs that the NBB is not notified in time when the donor passes away, probably as NBB asks for co-signing by a next of kin. Thus, the expectation is that the number of registered donors is almost equal to the number of autopsies that will take place in the future.

\section{Conclusions}

In conclusion, our program resulted in a steep increase in the number of registered prospective brain donors with psychiatric diagnoses. In 2010, 149 patients with a psychiatric disorder were registered at the NBB and in October 2016, this number had risen to 1149 and equaled the number of registrations of subjects with a neurological disease.

Our results provide evidence that, when starting a prospective brain donor program, one should aim at a faceto-face approach and a focus on older people, regardless of gender. The steep increase in psychiatric brain donors will result in a huge expansion of the amount of excellently phenotyped psychiatric brain tissue available for research, which will ultimately improve our understanding of the pathophysiology of psychiatric disorders and will create the possibility to develop better treatment strategies, resulting in a higher quality of life for people with psychiatric disorders.

\footnotetext{
Abbreviations

BEP/EMDR: 'Effectiveness and efficiency of Eye Movement Desensitization and Reprocessing therapy (EMDR) versus Brief Eclectic Psychotherapy (BEP) in the treatment of PTSD'; Booster: The effect of oxytocin on brain activity in police officers. Paroxetine/CGT = Randomized controlled trial 'the effectiveness of paroxetine versus trauma-focused cognitive behavioral therapy in the treatment of PTSD'; ADHD: Attention Deficit Hyperactivity Disorder; AMC DBS: Patients with OCD who underwent deep brain stimulation at the AMC; AMC regular: Patients who had a diagnostic intake or were treated for OCD at the Academic Medical Center in Amsterdam; ASD: Autism spectrum disorder; BD: Bipolar disorder; BiG: Bipolar Genetics; BioMap: The 'biological markers for PTSD' study; DIADE: Diagnostic Imaging of Affective Disorders using Emotion
}

Processing; ECT: Depressed patients treated with electroconvulsive therapy; GROUP: Genetic Risk and Outcome in Psychosis; MDD: Major depressive disorder; NBB-Psy: Netherlands Brain Bank for Psychiatry; NESDA: The Netherlands Study of Depression and Anxiety; NESDO: The Netherlands study of Depression in the Elderly; NOCDA: The Netherlands OCD Association Study: identifying risk factors for chronicity in the course of obsessive-compulsive disorder; OCD: Obsessive-compulsive disorder; PO: Police officers who were treated for PTSD at the Academic Medical Center; PTSD: Post-traumatic stress disorder; SCZ: Schizophrenia

\section{Acknowledgements}

We would like to thank all cohort participants for their unceasing dedication to the NBB-Psy project. Furthermore, we are grateful to the Netherlands Institute for Neuroscience and Royal Netherlands Academy of Arts and Sciences (KNAW) Amsterdam, the University Medical Center Utrecht Brain Center Rudolf Magnus, Academic Medical Center Amsterdam, Vrije Universiteit Amsterdam, Radboud University Medical Center Nijmegen and Erasmus Medical Center Rotterdam for the fruitful and pleasant collaboration. Finally, we acknowledge and thank Kim Alyousefi-van Dijk, Lisette Kuijper, Nel Hooiveld, Yvonne Kras, and Chantal Penseel for their dedication and efforts regarding the approach of cohort participants.

\section{Funding}

The Netherlands Organisation for Scientific Research (NWO) is the main funder of NBB-Psy, supplemented by funding of Royal Netherlands Academy of Arts and Sciences Amsterdam, University Medical Center Utrecht, Academic Medical Center Amsterdam, Free University Amsterdam, Radboud Medical Center Nijmegen and Erasmus Medical Center Rotterdam. Funding bodies had no role in the design of the study and collection, analysis, and interpretation of data and in writing the manuscript.

\section{Availability of data and materials}

The datasets used and/or analysed during the current study are available from the corresponding author on reasonable request.

\section{Authors' contributions}

GL made substantial contributions to conception and design, acquisition of data, and analysis and interpretation of data; and was a major contributor in writing the manuscript. MB was closely involved in the statistical analysis and interpretation of the data and was involved in revising the manuscript. MR and SP were closely involved in the study design, interpretation of data and revising the manuscript critically. All authors read and approved the final manuscript.

\section{Ethics approval and consent to participate}

The work of the Netherlands Brain Bank does not fall under the scope of the Medical Research Involving Human Subjects Act (WMO). Nonetheless, the NBB asked the Medical Research and Ethics Committee of the VU University Medical Center Amsterdam to review its procedures and documents (e.g. informed consent form: from participants who registered as brain donor, written informed consent was obtained). An official, written approval was given in April 2009. Furthermore, in October 2012 supplementary approval was given for the additional procedures for the NBB-Psy project.

\section{Consent for publication}

Not applicable.

\section{Competing interests}

The authors declare that they have no competing interests.

\section{Publisher's Note}

Springer Nature remains neutral with regard to jurisdictional claims in published maps and institutional affiliations.

Received: 15 February 2017 Accepted: 16 October 2017 Published online: 20 October 2017

\section{References}

1. Deep-Soboslay A, Benes FM, Haroutunian V, Ellis JK, Kleinman JE, Hyde TM. Psychiatric brain banking: three perspectives on current trends and future directions. Biol Psychiatry. 2011;69(2):104-12. 
2. Walker ER, McGee RE, Druss BG. Mortality in mental disorders and global disease burden implications: a systematic review and meta-analysis. JAMA psychiatry. 2015;72(4):334-41.

3. Whiteford HA, Ferrari AJ, Degenhardt L, Feigin V, Vos T. The global burden of mental, neurological and substance use disorders: an analysis from the global burden of disease study 2010. PLoS One. 2015;10(2):e0116820.

4. Austrom MG, Dickinson SLJ, Denny SS, Matthews BR, Gao S, Lu Y. Frontotemporal Dementia Caregivers and researchers: partnering for brain donation. American Journal of Alzheimer's Disease and Other Dementias. 2011;26(6):477-83.

5. Kaye JA, Dame A, Lehman S, Sexton G. Factors associated with brain donation among optimally healthy elderly people. J Gerontol. 1999;54A(11):M560-4.

6. Schmitt FA, Wetherby MM, Wekstein DR, Dearth CM, Markesbery WR. Brain donation in normal aging: procedures, motivations, and donor characteristics from the biologically resilient adults in neurological studies (BRAiNS) project. The Gerontologist. 2001;41(6):716-22.

7. Barnes $L L$, Shah RC, Aggarwal NT, Bennett AD, Schneider AJ. The minority aging research study: ongoing efforts to obtain brain donation in African Americans without dementia. Curr Alzheimer Res. 2012;9(6):734-45.

8. Shaw K, Gearing M, Davey A, Burgess M, Poon LW, Martin P, Green RC. Successful recruitment of centenarians for post-mortem brain donation: results from the Georgia centenarian study. The journal of bioscience and medicine. 2012;2(1):24

9. Beardsall L, Barkley C, O'Sullivan A. The response of elderly community residents to request for brain donation: an interim report. International Journal of Geriatric Psychiatry. 1992;7(3):199-202.

10. Garrick T, Howell S, Terwee P, Redenbach J, Blake H, Harper C. Brain donation for research: who donates and why? J Clin Neurosci. 2006;13(5):524-8.

11. Glaw XM, Garrick TM, Terwee PJ, Patching JR, Blake H, Harper C. Brain. Donation: who and why? Cell Tissue Bank. 2009;10(3):241-6.

\section{Submit your next manuscript to BioMed Central and we will help you at every step:}

- We accept pre-submission inquiries

- Our selector tool helps you to find the most relevant journal

- We provide round the clock customer support

- Convenient online submission

- Thorough peer review

- Inclusion in PubMed and all major indexing services

- Maximum visibility for your research

Submit your manuscript at www.biomedcentral.com/submit 